\title{
Current status of autologous stem cell transplantation for multiple myeloma
}

\author{
Rama Al Hamed ${ }^{1}$, Abdul Hamid Bazarbachi', Florent Malard (D)', Jean-Luc Harousseau ${ }^{2}$ and Mohamad Mohty ${ }^{1}$
}

\begin{abstract}
More than 30 years after its introduction, autologous stem cell transplantation (ASCT) remains the standard of care for young patients with newly diagnosed multiple myeloma. Not only did the arrival of novel agents such as immunomodulatory drugs (IMiDs), proteasome inhibitors (PI) and monoclonal antibodies not replace ASCT, instead they solidified its central role as standard of care. Novel agent use is now inarguably essential in induction, maintenance, and possibly consolidation. In light of these new advancements, new challenges arise in deciding on optimal practice. Who is most suited to undergo ASCT? Is there an age threshold that should not be surpassed? Should transplantation be embarked on early or is it reasonable to delay it? What are the optimal induction, consolidation, and maintenance therapies? What is the role of tandem transplantation in the era of novel agents and where do patient-specific cytogenetics come into the equation when deciding on treatment? These are some of the questions addressed in this review which we will attempt to answer with the latest currently available data.
\end{abstract}

\section{Introduction}

Multiple myeloma accounts for approximately $10 \%$ of hematologic cancers and $1 \%$ of all cancers in general ${ }^{1}$. Thirty years after the work of Powles, Barlogie, and McElwain led to the initiation of the concept of high-dose therapy (HDT) followed by autologous stem cell transplantation (ASCT) $)^{2-4}$, transplantation remains the standard for treating newly diagnosed multiple myeloma in young and in select, fit, elderly patients. The procedure's superiority was initially proven by the Intergroupe Francophone du Myeloma (IFM) and later confirmed by the UK Medical Research Council ${ }^{5-8}$. Despite its confirmed significant impact on event-free survival (EFS), multiple trials failed to depict significant impact on overall survival $(\mathrm{OS})^{9}$. Even though standard, the procedure is still challenged by inevitable relapses threatening long-term remissions, and is therefore challenged by some

\footnotetext{
Correspondence: Mohamad Mohty (mohamad.mohty@inserm.fr)

'Service d'hématologie clinique et thérapie cellulaire, Hôpital Saint-Antoine, INSERM UMRs 938 and université Sorbonne, Paris, France

2Institut de Cancerologie de I'Ouest, Centre René Gauducheau, Nantes-St Herblain, France

These authors contributed equally: Al Hamed Rama, Bazarbachi Abdul Hamid
}

myeloma experts who delay ASCT till relapse or progression $^{5,8}$.

The introduction of drugs such as thalidomide, lenalidomide, and bortezomib administered before and/or after HDT/ASCT gave way to the groundbreaking achievement of stringent complete response (sCR) with a normal kappa/lambda ratio (serum free light chain) ${ }^{10}$, immunophenotypic $\mathrm{CR}^{11}$, and molecular $\mathrm{CR}^{12}$, in addition to significantly increased $C R$ and $C R$ plus very good partial response rate $(\mathrm{VGPR})^{13,14}$. Thus, in the era of novel agents, embarking on transplantation is further scrutinized. Current ongoing studies are investigating incorporating different agents such as daratumumab and lenalidomide before and after transplantation, respectively. The challenge is thus to evaluate the necessity of HDT/ASCT when a powerful monoclonal antibody is combined with an induction regimen incorporating an immunomodulatory drug (IMiD) and a proteasome inhibitor (PI). This review addresses ten questions around the different steps of transplantation and lays forward current evidence from ongoing trials to aid decisionmaking. 


\section{Which patients are candidates for ASCT?}

There is no consensus regarding an age cutoff beyond which treatment with ASCT becomes questionable and as such, practice varies across institutions and countries. Generally, HDT/ASCT is reserved for patients younger than 65 years old with no severe comorbidities. Two important conditions are described below.

\section{Patient is over 65 years of age}

Most of the randomized studies have included patients younger than 65 years of age and so it becomes difficult to infer conclusions regarding this matter ${ }^{15}$. Usually, age of participants is limited to 65 years to avoid selection bias and limit toxicities and withdrawal from studies. However, this does not mean that ASCT is not feasible in older patients. On the contrary, it is in select patients ${ }^{16}$. A previous study whereby the median age of patients was 72 years old concluded that elderly multiple myeloma patients should not be excluded from transplantation displaying good results with melphalan $140 \mathrm{mg} / \mathrm{m}^{2}$ (ref. ${ }^{16}$ ). In the very few studies that did include older patients, melphalan doses were reduced $\left(100 \mathrm{mg} / \mathrm{m}^{2}\right.$ instead of $200 \mathrm{mg} / \mathrm{m}^{2}$ ) and the transplant procedure was repeated twice ${ }^{5}$. In a single center's experience, "young" patients (age range 30-65) who received high-dose melphalan (HDM/ASCT $\left(200 \mathrm{mg} / \mathrm{m}^{2}\right)$ ) and "elderly" patients (age range 66-75) who received two cycles of HDM/ ASCT $\left(100 \mathrm{mg} / \mathrm{m}^{2}\right)$ were compared ${ }^{17}$. The analysis demonstrated no significant difference in progression-free survival (PFS), OS, or treatment-related mortality between the two groups and among all subgroups ${ }^{17}$. Interestingly, PFS and OS in "elderly" patients appeared to improve after 2008, due to the increased incorporation of novel agents in the treatment, thus leading to the conclusion that the combination of ASCT and novel-based regimens were not subject to the influence of age on treatment outcome $^{17}$.

Currently, in the United States, fit patients up to 75 years old, receive $\mathrm{ASCT}^{16}$. On the other hand, in Europe, autologous transplants go up to the age of 70 offprotocol $^{18-20}$. The field of transplantation among elderly patients still lags behind and awaits randomized controlled trials (RCTs) to synthesize solid guidelines.

\section{Patient has renal impairment}

Renal impairment, per se, is not a contraindication to receiving HDT/ASCT. Nonetheless, it is a prompt reason to consider lower doses of therapy, as patients with renal impairment are more likely to suffer from HDM toxicities $^{21,22}$. Studies, including the DAUTOS observational study of the Polish myeloma study group, demonstrated that dialysis-dependent patients were more likely to develop toxicities and complications such as mucositis and infections, but had PFS and OS comparable to matched patients with normal renal function ${ }^{23,24}$. Also, the dose of melphalan mattered, with patients achieving better outcomes with $200 \mathrm{mg} / \mathrm{m}^{2}$ (ref. ${ }^{24}$ ). Interestingly, a proportion of patients were able to attain dialysisindependence after transplantation ${ }^{24}$. RCTs are yet to pave the way to guidelines regarding this transplantation scenario.

\section{What is the best induction treatment prior to ASCT?}

The role of induction chemotherapy prior to HDT/ASCT is to decrease tumor burden, thus deepening the response rate and increasing the likelihood of engraftment, while retaining the maximum possible tolerability and minimum possible toxicity on normal hematopoietic cells. As a result, and prior to the introduction of novel agents, alkylating agents were avoided during induction, and regimens were dexamethasone-based such as the VAD regimen (vincristine, doxorubicin and dexamethasone) ${ }^{1}$. With the advent of new drugs, multiple trials have proven the superiority of induction regimens containing one or two novel agents (thalidomide or bortezomib) over the VAD regimen in increasing $\mathrm{CR}, \mathrm{CR}$ plus near-complete response (nCR), or VGPR rates pre- and post ASCT ${ }^{25-28}$. Trials that compared two-drug (TD: thalidomide-dexamethasone or VD: bortezomib-dexamethasone) to three-drug induction (VTD: bortezomib, thalidomide, dexamethasone) have proven supremacy of the latter combination ${ }^{13,29,30}$. VTD was also proven superior to bortezomib, cyclophosphamide, and dexamethasone (VCD), thus highlighting the synergistic effect of combining an IMiD with bortezomib and dexamethasone ${ }^{31}$. As such, VTD became a standard induction regimen, whereby the role of a PI such as bortezomib is irreplaceable due to its demonstrated usefulness in high-risk patients ${ }^{26,32,33}$. Furthermore, although the general practice is to use 3-4 cycles of VTD before transplant, the use of 6 cycles of VTD was associated with deeper responses. This is to be weighed against increased side effects, specifically neuropathy, upon administering 6 cycles instead of 3-4 (ref. ${ }^{30}$ ).

Similarly, the two-drug regimen, lenalidomide and dexamethasone (RD), was compared to bortezomib, lenalidomide, and dexamethasone (VRD) whereby VRD resulted in significantly increased PFS, response duration, and OS resulting in the IFM introducing VRD as induction $^{8,34,35}$. In addition, the PETHEMA/GEM trial investigated induction with VRD-GEM with full dose lenalidomide from days 1 to 21, demonstrating an ORR of $85 \%$ post induction and $58 \%$ of patients achieving MRDnegativity post consolidation ${ }^{36}$.

Daratumumab (DARA), an anti-CD38+ monoclonal antibody, has been evaluated in patients with refractory disease $^{37,38}$. The Cassiopeia phase III trial and the Griffin phase II trial compare DARA-VTD to VTD and 


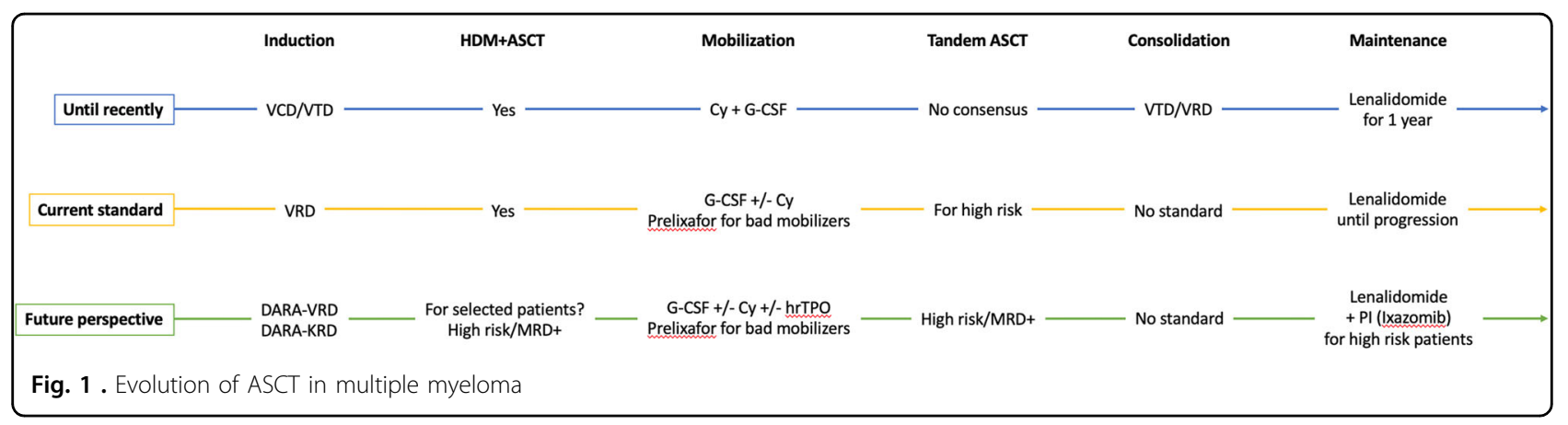

DARA-VRD to VRD, respectively, demonstrating hopeful results of adding daratumumab ${ }^{39-41}$. Daratumumab plus cyclophosphamide, bortezomib, and dexamethasone (Dara-CyBorD) during induction was investigated in the phase II Lyra trial. Recent updates of the trial demonstrate activity and tolerability of Dara-CyBorD irrespective of high-risk cytogenetics with 12-month PFS and OS rates of $87 \%$ and $99 \%$, respectively ${ }^{42}$. Finally, daratumumab is also being combined with carfilzomib, lenalidomide, and dexamethasone (KRD) in a phase Ib trial, whereby the combined regimen yielded 100\% ORR, 91\% $\geq$ VGPR, and $43 \% \geq C R$, with no negative impact on stem cell harvesting while retaining consistency of the DARA-KRD safety profile $^{43}$.

MRD negativity, defined as the absence of disease within one million bone marrow cells, has been examined due to its important prognostic value at different stages of the transplantation process. The depth of response after induction and before ASCT determines patients' prognoses after ASCT since the quality of response post induction and prior to ASCT are predictive of long-term PFS post ASCT ${ }^{13,33,44-49}$. The final analysis of the IFM2009 prospective trial demonstrated the significance of MRD negativity, whereby patients achieving MRD negativity after induction with VRD had a similar OS irrespective of whether they received an ASCT or not ${ }^{50}$. In addition, MRD negativity proved to be a more powerful predictor of outcome than cytogenetics, whereby patients with high-risk cytogenetics who achieved MRD negativity had better outcomes than patients with standard-risk cytogenetics who did not ${ }^{50}$. This could mean that MRD could potentially become essential in stratifying patients during maintenance and consolidation randomization and when deciding on maintenance duration ${ }^{50}$.

With no evidence that four-drug regimens (IMiD, PI, alkylating agent, and steroid) are superior ${ }^{51,52}$, VTD and VRD remain the most currently used pre-transplant induction regimens, awaiting the results of ongoing trials testing the efficacy of adding daratumumab or the possible substitution of bortezomib with carfilzomib, which has been found to be safe and well tolerated with exceptional response rates (Fig. 1) ${ }^{53,54}$.

\section{What is the best stem cell mobilization procedure prior to ASCT?}

A critical and essential step prior to ASCT is mobilization of hematopoietic stem cells (HSC) from the bone marrow to be harvested in peripheral blood. The minimum CD34+ stem cell dose considered sufficient for successful engraftment is $2 \times 10^{6}$ cells CD $34+/ \mathrm{kg}$, but the optimal target is usually set at $5 \times 10^{6} \mathrm{CD} 34+/ \mathrm{kg}$. This can be done by steady-state mobilization with granulocyte colony-stimulating factors $(\mathrm{G}-\mathrm{CSF})^{55}$ or chemomobilization by the addition of chemotherapeutic agent $(\mathrm{s})^{5}$. Currently, two G-CSF cytokines are approved for the mobilization of autologous HSC: filgrastim $(10 \mu \mathrm{g} / \mathrm{kg} /$ day for 4-6 consecutive days and apheresis on days 5 or 6 ) and lenograstim $(10 \mu \mathrm{g} / \mathrm{kg} /$ day for $4-6$ days and apheresis between days 5 and 7$)^{5}$. Even though tolerable, the use of G-CSF cytokines can yield suboptimal peripheral HSC harvest ${ }^{56}$. The most commonly used agent for chemomobilization is high-dose cyclophosphamide (2-4g/ $\left.\mathrm{m}^{2}\right)^{57}$, followed by filgrastim or lenograstim $(5 \mu \mathrm{g} / \mathrm{kg} /$ day 1-5 days after completion of chemotherapy till last apheresis) ${ }^{5}$. This strategy can potentially decrease tumor burden, but the time to peripheral blood stem cell (PBSC) harvest is prolonged, with increased side effects ${ }^{5}$. Since some patients fail to mobilize $e^{58-60}$, the addition of new mobilization agents such as plerixafor, a chemokinereceptor 4 (CXCR4) antagonist, enhanced the stem cell mobilization effect of G-CSF ${ }^{56,61}$. Even though proven to be highly effective, plerixafor is not widely available ${ }^{62}$.

Other studies have compared cyclophosphamide to mCVAD (modified cyclophosphamide, vincristine, doxorubicin and dexamethasone) and mCBAD (modified cyclophosphamide, bortezomib, doxorubicin, and dexamethasone) concluding that more intense regimens are not superior to cyclophosphamide alone in mobilization $^{63}$. A randomized phase III trial compared mobilization with cytarabine (Ara-C) and G-CSF versus G-CSF alone demonstrating improved yields with the addition of Ara-C, but increased hematologic toxicities ${ }^{64}$. It is noteworthy that employing lenalidomide in induction has been found to be one of the factors compromising stem cell mobilization success ${ }^{65,66}$, mainly due to lenalidomide 
upregulating CXCR4 and increasing the binding of stem cells to the stroma ${ }^{67}$. A prospective randomized phase II sub-study in the Finnish Myeloma Study Group-MM02 trial compared low-dose cyclophosphamide $\left(2 \mathrm{mg} / \mathrm{m}^{2}\right)$ plus G-CSF to G-CSF alone for mobilization in patients who have received lenalidomide during induction ${ }^{68}$. The addition of cyclophosphamide to G-CSF was superior, although G-CSF could yield similar results in patients receiving no more than three cycles of $\mathrm{VRD}^{68}$. In addition, the addition of plerixafor for mobilization in patients who received lenalidomide has also been proven effective ${ }^{69}$. As such, with the availability of plerixafor, the prolonged use of lenalidomide does not hinder stem cell mobilization. Finally, combining human recombinant thrombopoietin (hrTPO) to G-CSF and cyclophosphamide improved yields compared to cyclophosphamide and G-CSF alone ${ }^{70}$.

Therefore, the most currently used regimens are either chemo-mobilization with high-dose cyclophosphamide plus G-CSF or steady-state mobilization alone, with preemptive use of plerixafor when suboptimal mobilization is predicted by a low circulating CD34 count.

\section{What is the optimal conditioning regimen prior to ASCT?}

The current accepted standard for HDT is intravenous high-dose melphalan $\left(200 \mathrm{mg} / \mathrm{m}^{2}\right)$. Previous trials attempting to replace this with oral and intravenous busulfan have failed, due to increased toxicity and lack of superiority, respectively ${ }^{71,72}$. The effect of intravenous busulfan is being studied in a phase III trial whereby $\mathrm{HDM}$ is compared to busulfan-melphalan (Bu-Mel: busulfan $130 \mathrm{mg} / \mathrm{m}^{2}$ daily for 4 days followed by two daily doses of melphalan at $\left.70 \mathrm{mg} / \mathrm{m}^{2}\right)^{73}$. The trial has demonstrated increased PFS with $\mathrm{Bu}-\mathrm{Mel}$ without a significant difference in response rates ${ }^{73}$. Higher doses of melphalan $\left(>200 \mathrm{mg} / \mathrm{m}^{2}\right)$, which proved useful for patients with primary refractive or relapsing disease nearly 20 years ago $^{74}$, are also being investigated. A randomized study comparing conditioning with melphalan 280 to $200 \mathrm{mg} / \mathrm{m}^{2}$ while receiving amifostine demonstrated significantly higher ORR and $\mathrm{nCR}$ without an improvement in OS and PFS in patients receiving melphalan $280 \mathrm{mg} / \mathrm{m}^{2}$ at the cost of higher incidence of grade 2-3 mucositis and gastrointestinal toxicities ${ }^{75}$. Another study demonstrated similar results with deeper responses on melphalan $280 \mathrm{mg} / \mathrm{m}^{2}$ without translating into improved survival ${ }^{76}$.

Bortezomib's effect in transplant conditioning was investigated when combined with HDM in the IFM 201402 phase III study ${ }^{77}$. This showed no superiority of Bortezomib-HDM over HDM alone in terms of response rate, $\mathrm{OS}$ or $\mathrm{PFS}^{77}$. In addition, the role of bendamustine added to melphalan as part of conditioning is being explored, highlighting improved response rates and $\mathrm{PFS}^{78,79}$.
As such, HDM remains the standard conditioning regimen prior to ASCT awaiting results of clinical trials of other conditioning regimens (if any).

\section{What is the impact of consolidation therapy after ASCT?}

The aim of short-term consolidation therapy after HDT/ASCT is to improve disease response with limited toxicity. Incorporating consolidation therapy in patients with a good response after ASCT was found to increase the $\mathrm{CR}$ rate and molecular remission, thus prolonging $\mathrm{PFS}^{12}$. The Italian Myeloma study group has previously investigated the effect of VTD versus TD as induction therapy before and as consolidation therapy after double ASCT, demonstrating VTD's superior influence on CR/ $\mathrm{nCR}$ rates and $\mathrm{PFS}^{13}$. Similarly, VRD proved superiority in consolidation ${ }^{35}$. These trials were very encouraging; nonetheless, randomized trials were needed to prove impact.

The second randomization in the EMN02/HO95 trial compared the aftermaths of receiving two cycles of VRD consolidation followed by lenalidomide versus lenalidomide maintenance alone, demonstrating the significant advantage VRD consolidation inferred in prolonging $\mathrm{PFS}^{80}$. Moreover, PFS was prolonged in most of the predefined groups in the study including ISS I and II, low-risk cytogenetics, irrespective of whether patients received VMP (bortezomib, melphalan, and prednisone) or transplantation prior to consolidation ${ }^{80}$. Nonetheless, VRD consolidation failed to improve PFS in patients with highrisk cytogenetics ((del17p and/or $t(4 ; 14)$ and/or $t$ $(14 ; 16))^{80}$. This confirms the benefit of VRD consolidation followed by lenalidomide maintenance in younger, newly diagnosed multiple myeloma patients with low-risk disease $^{80}$.

Along the same line of the EMN02/HO95 trial, the StaMINA phase III trial randomized patients to compare HDM/ASCT plus VRD consolidation plus lenalidomide maintenance, versus tandem HDM/ASCT plus lenalidomide maintenance, versus single HDM/ASCT plus lenalidomide maintenance ${ }^{81}$. It concluded that the addition of VRD consolidation or a tandem ASCT was not superior to standard ASCT followed by lenalidomide in upfront treatment of newly diagnosed multiple myeloma ${ }^{81}$.

With the currently available data, the role of posttransplant consolidation remains controversial.

\section{What is the impact of maintenance therapy after ASCT?}

Even though HDT/ASCT is the standard frontline treatment for newly diagnosed multiple myeloma patients, ASCT is not curative, and progressions and relapses are common even if $\mathrm{CR}$ is attained posttransplant $t^{6,7,82}$. Maintenance therapy is thus added and 
is expected to be gentle with the safest profile post ASCT, but unlike consolidation, it is administered long-term to deepen the response, prevent progression, and prolong $\mathrm{OS}^{5}$.

Thalidomide, having already been used in different myeloma treatment settings and being an oral agent, has been tested in several randomized trials, most of which demonstrated benefit in terms of response rates but not $\mathrm{OS}^{83-85}$. Thalidomide was repeatedly associated with peripheral neuropathy, fatigue, and other side effects, all of which resulted in patient-reported decreased quality of life despite prolonged duration of disease control ${ }^{85}$. Thus, when used in the maintenance setting, the dosage and duration should be limited to $100 \mathrm{mg}$ daily and 6-12 months, respectively, as suggested by Spencer et al.'s study ${ }^{86}$.

Lenalidomide maintenance has been shown to be well tolerated and to dramatically improve PFS and OS (Table 1$)^{87,88}$. A recent meta-analysis of three RCTs, CALGB, IFM, and GIMEMA, that compared lenalidomide maintenance to placebo or observation, has demonstrated clinically valuable results ${ }^{89}$. Lenalidomide significantly improved PFS in all subgroups of patients regardless of age, myeloma severity and staging, and induction regimen (52.8 versus 23.5 months), even though patients who had received lenalidomide in induction, or had achieved a deeper response post-transplant, were more likely to benefit from lenalidomide ${ }^{89}$. OS was also significantly improved in the lenalidomide arm, except in women older than 60 years with poor cytogenetics ${ }^{89}$. Overall, the addition of lenalidomide reduced the chance of death by a substantial $25 \%$, thus increasing median survival by approximately 2.4 years ${ }^{89}$. As demonstrated in previous studies, an increased incidence of second primary malignancies, albeit modest, was associated with lenalidomide, though the time to death due to a second primary malignancy did not differ between the two groups $^{89}$. Such results propose lenalidomide as a standard maintenance drug in transplant-eligible patients ${ }^{89}$. Recent updates of the Myeloma XI trial's results were in concordance with the meta-analysis ${ }^{90}$.

So far in previous trials, lenalidomide has been given in low doses until progression or adverse events develop, and this practice is currently approved by both, FDA and EMA. Given that $30 \%$ of cases with premature termination of lenalidomide were attributed to toxicities and second primary malignancies ${ }^{89}$, the question that remains is regarding the optimal duration of treatment with lenalidomide for safety and cost.

Finally, bortezomib was also tested as part of maintenance, either alone or in combination with IMiDs, demonstrating improved PFS, but not $\mathrm{OS}^{28}$. Nonetheless, bortezomib poses an obstacle due to its subcutaneous/i.v. administration. The first oral PI, ixazomib, is currently being investigated. So far, it appears to have positive effects, with a safety profile comparable to that of lenalidomide alone, and is manageable by dose reductions (Fig. 1) ${ }^{91}$. Ixazomib was also compared to placebo in the multicenter TOURMALINE-MM3 trial with a median follow-up of 31 months, whereby there was a 39\% improvement in PFS and a $28 \%$ reduction in progression or death. Ixazomib also allowed for deeper responses to be achieved $^{92}$.

\section{What is the value of single versus tandem ASCT?}

In the 1990s, in an attempt to improve survival and overall outcome, the concept of tandem transplant came about in an era where conventional chemotherapy was the only available drug $^{93}$. Previous randomized trials had demonstrated improved outcomes with tandem transplantation in terms of PFS and OS even in patients who had not achieved a VGPR after the first transplant ${ }^{1,94}$. An alternative treatment approach, total therapy 3 (TT3), including induction, tandem ASCT, consolidation, and maintenance, has allowed one of the best results to be achieved $(\mathrm{CR} / \mathrm{nCR}$ rate of $83 \%$, 2-year PFS of $84 \%$, and 2year OS of $86 \%)^{95}$.

Table 1 Lenalidomide maintenance trials

\begin{tabular}{|c|c|c|c|c|c|}
\hline Study & Median follow-up & $N$ & Treatment & Outcome & \\
\hline Meta-analysis & 79.5 months & & & PFS & OS \\
\hline IFM & & 605 & Lenalidomide & 52.8 months & Median OS not reached \\
\hline CALGB & & 603 & Placebo/Observation & 23.5 months & 86 months \\
\hline GIMEMA & & & & (HR 0.48; 95\% Cl 0.41-0.55) & (HR 0.75; 95\% Cl 0.63-0.9) \\
\hline \multirow[t]{4}{*}{ Myeloma XI } & 28.7 months & & & PFS & \\
\hline & & 1136 & Lenalidomide & 60.3 months & \\
\hline & & 834 & Observation & 30.1 months & \\
\hline & & & & (HR 0.47; 95\% Cl 0.39-0.57) & \\
\hline
\end{tabular}


Long-term analysis of the GMMG-HD2 trial compared single versus tandem transplantation with conditioning with melphalan $\left(200 \mathrm{mg} / \mathrm{m}^{2}\right)^{96}$. The study proved the non-inferiority of single transplantation compared to tandem in the sense that OS and EFS did not significantly differ ${ }^{96}$. Nonetheless, the $\mathrm{CR}$ rates were significantly improved after the second transplantation ${ }^{96}$. Due to high drop-out rates, lack of use of novel therapy, and lack of subgroup analysis, the results of this study are to be cautiously interpreted ${ }^{96}$.

In the era of novel drugs, we needed trials to evaluate the impact of tandem transplantation such as the EMN02/ HO95 and StaMINA trials ${ }^{81,97}$. The EMN02/HO95 trial explored the result of tandem versus single transplantation in newly diagnosed multiple myeloma patients ${ }^{97}$. Tandem transplantation was shown to improve the depth of the response by $25 \%$ with more than $50 \%$ of the patients achieving at least a $\mathrm{CR}^{97}$. PFS and OS were significantly improved after a second transplant, with approximately $30 \%$ reduction in the risk of death and progression $^{97}$. Updated results of the EMN02/HO95 confirmed the improved 3-year PFS from 63 months after one ASCT to 73.1 after two ASCTs ${ }^{98}$. Importantly, the positive effect of tandem ASCT was seen in high-risk groups, in which randomization to receive double ASCT was found to be an independent predictor of $\mathrm{PFS}^{97,98}$. The analysis thus concluded that double frontline ASCT was superior to single ASCT in terms of PFS and OS in all patients, including poor prognosis subgroups, indicating that the latter were the most likely to benefit ${ }^{97,98}$.

On the other hand, the StaMINA trial failed to show superiority of tandem versus single transplant in the era of novel agents ${ }^{81}$. It is noteworthy that more than $30 \%$ of patients randomized to tandem transplant did not receive the second transplant ${ }^{81}$.

Overall, with the currently available data, a second ASCT may be beneficial in high-risk patients including patients with high-risk cytogenetics and RISS 3 category of disease.

\section{What is the added value of HDT/ASCT in the era of triple novel agent regimens?}

With the advent of novel agents, it becomes questionable whether or not HDT/ASCT has any added value at all. The previously mentioned SWOG S0777 trial compared outcomes of lenalidomide and dexamethasone alone (RD) to bortezomib, lenalidomide, and dexamethasone (VRD) without an intent to transplant ${ }^{34}$. The results confirmed the superiority of VRD in increasing PFS, response duration, and $\mathrm{OS}^{34}$. As such, it is suggested that VRD alone is not only safe but has comparable PFS/ OS to HDT/ASCT. Trials were thus necessary to compare novel agents in combination to ASCT to novel agents alone.
A randomized phase III trial for the IFM2009 was conducted to compare the efficacy of combination therapy with lenalidomide, bortezomib, and dexamethasone (RVD) alone to RVD plus HDT/ASCT in newly diagnosed multiple myeloma patients younger than 65 years old ${ }^{8}$. Patients were randomized so as to receive induction therapy with three cycles of RVD, and then consolidation with either five more cycles of RVD or high-dose melphalan followed by ASCT and two cycles of $\mathrm{RVD}^{8}$. All patients received lenalidomide maintenance for 1 year $^{8}$. The use of transplantation in addition to novel agents as opposed to RVD alone resulted in significant improvement in PFS (50 versus 36 months, adjusted HR 0.65), CR rate (59\% versus $48 \%$ ), MRD negativity (79\% versus $65 \%$ ), and median time to disease progression (50 versus 36 months), with no advantage regarding $\mathrm{OS}^{8}$. In the phase III EMN02/ HO95 study mentioned earlier, the first randomization compared the outcomes of HDT/ASCT (single or double) versus bortezomib-melphalan-prednisone (VMP) after induction with $\mathrm{VCD}^{97}$. Even though bortezomib has been repeatedly shown to increase PFS and OS, upfront ASCT was associated with decreased risk of progression and death and improved 3-year PFS irrespective of initial prognostic factors ${ }^{97}$.

On the other hand, two studies whereby transplantation was compared to alkylating agent-based regimens and lenalidomide associated a survival benefit with first-line transplantation $^{99,100}$. Nonetheless, these trials did not incorporate bortezomib in their non-transplant arm which could explain the improved $\mathrm{OS}^{99,100}$.

The extent of improved PFS in the transplant arm in both EMN02/HO95 and IFM2009 trials, likely attributed to a deeper response through increased CR and MRD rates, suggest that given more observational time, we could possibly find an improvement in OS as was the case for lenalidomide maintenance. This is especially relevant given that relapsed patients receive comparable treatment including a second ASCT and the use of newly introduced agents.

The next challenge is to evaluate the necessity of HDT/ ASCT when a monoclonal antibody such as daratumumab is added to a powerful induction regimen combining an IMiD and a PI, and whether this strategy can cure a fraction of patients. As such, we conclude that ASCT remains first line even in the era of novel agents. The impending challenge remains whether or not transplantation will be later substituted by less intensive novel agent combinations.

\section{What is the value of early versus late ASCT?}

Frontline HDT/ASCT has been the standard for treating newly diagnosed multiple myeloma in young, fit patients and select elderly patients. Nonetheless, with the advent of present novel therapies, specialists have 
challenged the notion that HDT/ASCT should be administered early after diagnosis.

In 1998 before the era of novel agents, Fermand et al. ${ }^{101}$ studied the effect of autologous transplantation timing (early versus late) on OS. Patients who were randomized into the "early" arm received HDT/ASCT right away and those in the "late" arm received conventional chemotherapy until progression or relapse whereby they were supported with HDT/ASCT as well ${ }^{101}$. There was no difference in OS between the two groups ${ }^{101}$. Time without symptoms, treatment and treatment toxicity (TWiST) was also evaluated whereby the period spent without chemotherapy was longer in patients who received early HDT/ASCT, suggesting a clinical benefit of early versus late transplantation ${ }^{101}$. Several retrospective trials failed to demonstrate benefits in OS when comparing early to late HDT/ASCT, which could be attributed to selection bias regarding patients in the "late" group ${ }^{102}$.

As previously mentioned for the IFM2009 trial, comparing VRD to VRD plus transplant yielded significantly better outcome with upfront ASCT in terms of CR rate, PFS, and MRD negativity. This highlights that, even in light of novel agents which have already been proven to drastically improve treatment outcomes, transplantation could further improve results. Nonetheless, OS was not affected by ASCT taking into account that transplantation was only done in two-third of the cases due to age, progression, and comorbidities, indicating that the benefits of upfront ASCT can be weighed against the toxicities of chemotherapy and transplantation, especially since late transplantation could secure a similar OS to early transplantation ${ }^{8}$. As such, in the absence of improvement in OS, delayed ASCT could be an option.

In the phase III EMN02/HO95 study mentioned earlier, the first randomization compared the outcomes of HDT/ ASCT (single or double) versus VMP after induction with $\mathrm{VCD}^{97}$. Even though bortezomib has been repeatedly shown to increase PFS and OS, upfront ASCT was associated with a $24 \%$ reduction in risk of progression or death $^{97}$. The estimated 3-year PFS was also significantly higher with upfront ASCT, regardless of the presence of poor prognostic factors ${ }^{97}$.

As such, it is safe to conclude that ASCT can improve outcomes whether performed as first line or as a rescue treatment $^{1,101}$. Therefore, frontline ASCT remains the standard of treatment for fit, young and select elderly patients with newly diagnosed multiple myeloma.

\section{What is the role of ASCT as salvage therapy?}

Salvage therapy is defined as ASCT given to a patient with signs of disease progression after an earlier ASCT ${ }^{103}$. By the BSBMT/UKMF Myeloma X trial, salvage ASCT with $200 \mathrm{mg} / \mathrm{m}^{2}$ melphalan was superior to cyclophosphamide $400 \mathrm{mg} / \mathrm{m}^{2}$ weekly for 12 weeks upon relapse and re-induction with $\mathrm{VAD}^{104}$. The time to disease progression (19 versus 11 months) and OS (67 versus 52 months) were significantly in favor of salvage ASCT $^{104}$. As such, ASCT can be considered for salvage in fit patients if the interval between the first ASCT and relapse is 18 months or more ${ }^{5,105}$. This awaits trials that compare salvage ASCT with novel agents including the German study by Goldschmidt et al. comparing salvage ASCT to lenalidomide/dexamethasone.

\section{Conclusion and future prospects}

Up until today, 30 years after its introduction, HDT/ ASCT remains the standard of care for patients with newly diagnosed multiple myeloma. Despite the advent of novel agents, ASCT remains a very common treatment modality, especially in Europe and is included in all ongoing and proposed trials. The latter is yet to be challenged by many novel agents (including earlier use of CAR $\mathrm{T}$ cells) which are continuously explored. As such, and by projecting the results of the ALCYONE trial which proved superiority of DARA-VMP over VMP in transplantineligible patients ${ }^{106}$, we conclude that there appears to be a place for daratumumab in future induction regimens, due to its promising preliminary additive effect to triple regimens in terms of response. Multiple trials are currently looking into daratumumab in transplant-ineligible patients with recently released updates, suggesting that the addition of daratumumab allows deeper response, increased PFS, and improved MRD negativity ${ }^{107}$. Such results could be possibly extrapolated to the subset of patients who are newly diagnosed and are transplant eligible. Since melphalan, a cornerstone in conditioning prior to ASCT, is usually given at a fixed dose, patients can either reach concentrations that are above the median, thus sustaining survival but suffering increased toxicity or they can fail to reach median concentrations and suffer from untreated disease. To overcome that, pharmacokinetic (PK)-directed melphalan dosing is looked into as a potential means to determine optimal dosing in individual patients. Whether novel melphalan formulations such as FDA-approved propylene glycol-free melphalan have increased efficacy and decreased toxicity compared to melphalan is to be investigated. One study failed to demonstrate superiority of propylene glycol-free melphalan over melphalan ${ }^{108}$. Whether or not to embark on early transplantation is yet to be investigated; although it has been made clear that ASCT is synergistic to triple novel agent-based therapy, it could be delayed when toxicities outweigh benefits. When dealing with unfavorable cytogenetics and poor prognostic factors, tandem transplantation appears to be an encouraging strategy. Whereas the use of post-transplant consolidation is controversial, lenalidomide maintenance prolongs PFS and OS and can be considered as a standard of care. One 
important endpoint to be measured in future studies is MRD negativity in standard or high-risk disease, since it is a significant predictor of PFS, OS, and potential cure in a fraction of patients ${ }^{109}$. The objective is thus to achieve MRD negativity $\left(<10^{-6}\right)$, a predictor of better outcome ${ }^{110}$. To possibly achieve MRD negativity at even lower cutoff, daratumumab will likely need to be added. Finally, even though first line for multiple myeloma treatment, the use of transplantation remains limited by accessibility and availability which differs across nations and is limited in developing countries.

\section{Conflict of interest}

M.M. discloses lectures honoraria and research support from Amgen, Celgene, Janssen, Sanofi, and Takeda whose products are discussed in this work.

\section{Publisher's note}

Springer Nature remains neutral with regard to jurisdictional claims in published maps and institutional affiliations.

Received: 12 January 2019 Revised: 5 March 2019 Accepted: 12 March 2019 Published online: 08 April 2019

\section{References}

1. Harousseau, J. L. \& Moreau, P. Autologous hematopoietic stem-cell transplantation for multiple myeloma. N. Engl. J. Med. 360, 2645-2654 (2009).

2. McElwain, T. J. \& Powles, R. L. High-dose intravenous melphalan for plasmacell leukaemia and myeloma. Lancet 2, 822-824 (1983).

3. Barlogie, B., Hall, R., Zander, A., Dicke, K. \& Alexanian, R. High-dose melphalan with autologous bone marrow transplantation for multiple myeloma. Blood 67, 1298-1301 (1986)

4. Barlogie, B. et al. High-dose chemoradiotherapy and autologous bone marrow transplantation for resistant multiple myeloma. Blood 70, 869-872 (1987).

5. Mohty, M. \& Harousseau, J. L. Treatment of autologous stem cell transplanteligible multiple myeloma patients: ten questions and answers. Haematologica 99, 408-416 (2014).

6. Attal, M. et al. A prospective, randomized trial of autologous bone marrow transplantation and chemotherapy in multiple myeloma. Intergroupe Francais du Myelome. N. Engl. J. Med. 335, $91-97$ (1996).

7. Child, J. A. et al. High-dose chemotherapy with hematopoietic stem-cell rescue for multiple myeloma. N. Engl. J. Med. 348, 1875-1883 (2003).

8. Attal, M. et al. Lenalidomide, Bortezomib, and Dexamethasone with transplantation for myeloma. N. Engl. J. Med. 376, 1311-1320 (2017).

9. Koreth, J. et al. High-dose therapy with single autologous transplantation versus chemotherapy for newly diagnosed multiple myeloma: a systematic review and meta-analysis of randomized controlled trials. Biol. Blood Marrow Transplant. 13, 183-196 (2007).

10. Barlogie, B., van Rhee, F., Shaughnessy, J. D. Jr, Anaissie, E. \& Crowley, J. Making progress in treating multiple myeloma with total therapies: issue of complete remission and more. Leukemia 22, 1633-1636 (2008).

11. Paiva, B. et al. Comparison of immunofixation, serum free light chain, and immunophenotyping for response evaluation and prognostication in multiple myeloma. J. Clin. Oncol. 29, 1627-1633 (2011).

12. Ladetto, M. et al. Major tumor shrinking and persistent molecular remissions after consolidation with bortezomib, thalidomide, and dexamethasone in patients with autografted myeloma. J. Clin. Oncol. 28, 2077-2084 (2010).

13. Cavo, M. et al. Bortezomib with thalidomide plus dexamethasone compared with thalidomide plus dexamethasone as induction therapy before, and consolidation therapy after, double autologous stem-cell transplantation in newly diagnosed multiple myeloma: a randomised phase 3 study. Lancet 376, 2075-2085 (2010).
14. Moreau, P., Avet-Loiseau, H., Harousseau, J. L. \& Attal, M. Current trends in autologous stem-cell transplantation for myeloma in the era of novel therapies. J. Clin. Oncol. 29, 1898-1906 (2011).

15. Facon, T. et al. Melphalan and prednisone plus thalidomide versus melphalan and prednisone alone or reduced-intensity autologous stem cell transplantation in elderly patients with multiple myeloma (IFM 99-06): a randomised trial. Lancet 370, 1209-1218 (2007).

16. Badros, A. et al. Autologous stem cell transplantation in elderly multiple myeloma patients over the age of 70 years. Br. J. Haematol. 114, 600-607 (2001).

17. Judith Neukirchen, P. A. et al. Favourable outcome of elderly patients with multiple myeloma treated with tandem melphalan 100 high-dose therapy autologous stem cell transplantation and novel agents-a single center experience. Blood 128, 3460 (2016).

18. Palumbo, A. et al. Dose-intensive melphalan with stem cell support (MEL 100) is superior to standard treatment in elderly myeloma patients. Blood $\mathbf{9 4}$, 1248-1253 (1999).

19. Gay, F. et al. Bortezomib induction, reduced-intensity transplantation, and lenalidomide consolidation-maintenance for myeloma: updated results. Blood 122, 1376-1383 (2013).

20. Straka, C. et al. Autotransplant with and without induction chemotherapy in older multiple myeloma patients: long-term outcome of a randomized trial. Haematologica 101, 1398-1406 (2016)

21. Badros, A. et al. Results of autologous stem cell transplant in multiple myeloma patients with renal failure. Br. J. Haematol. 114, 822-829 (2001)

22. Tosi, P. et al. Safety of autologous hematopoietic stem cell transplantation in patients with multiple myeloma and chronic renal failure. Leukemia 14, 1310-1313 (2000)

23. Waszczuk-Gajda, A. et al. Autologous peripheral blood stem cell transplantation in dialysis-dependent multiple myeloma patients-DAUTOS Study of the Polish Myeloma Study Group. Eur. J. Haematol. 101, 475-485 (2018).

24. Mahindra, A. et al. Autologous hematopoietic cell transplantation for multiple myeloma patients with renal insufficiency: a center for international blood and marrow transplant research analysis. Bone Marrow Transplant. 52 1616-1622 (2017)

25. Lokhorst, H. M. et al. A randomized phase 3 study on the effect of thalidomide combined with adriamycin, dexamethasone, and high-dose melphalan, followed by thalidomide maintenance in patients with multiple myeloma. Blood 115, 1113-1120 (2010).

26. Harousseau, J. L. et al. Bortezomib plus dexamethasone is superior to vincristine plus doxorubicin plus dexamethasone as induction treatment prior to autologous stem-cell transplantation in newly diagnosed multiple myeloma: results of the IFM 2005-01 phase III trial. J. Clin. Oncol. 28, 4621-4629 (2010).

27. Morgan, G. J. et al. Cyclophosphamide, thalidomide, and dexamethasone as induction therapy for newly diagnosed multiple myeloma patients destined for autologous stem-cell transplantation: MRC Myeloma IX randomized trial results. Haematologica 97, 442-450 (2012).

28. Sonneveld, P. et al. Bortezomib induction and maintenance treatment in patients with newly diagnosed multiple myeloma: results of the randomized phase III HOVON-65/ GMMG-HD4 trial. J. Clin. Oncol. 30, 2946-2955 (2012).

29. Moreau, P. et al. Bortezomib plus dexamethasone versus reduced-dose bortezomib, thalidomide plus dexamethasone as induction treatment before autologous stem cell transplantation in newly diagnosed multiple myeloma. Blood 118, 5752-5758 (2011). quiz 982.

30. Rosinol, L. et al. Superiority of bortezomib, thalidomide, and dexamethasone (VTD) as induction pretransplantation therapy in multiple myeloma: a randomized phase 3 PETHEMA/GEM study. Blood 120, 1589-1596 (2012).

31. Moreau, P. et al. VTD is superior to VCD prior to intensive therapy in multiple myeloma: results of the prospective IFM2013-04 trial. Blood 127, 2569-2574 (2016).

32. Avet-Loiseau, $\mathrm{H}$. et al. Bortezomib plus dexamethasone induction improves outcome of patients with $t(4 ; 14)$ myeloma but not outcome of patients with del(17p). J. Clin. Oncol. 28, 4630-4634 (2010).

33. Sonneveld, P. et al. Bortezomib-based versus nonbortezomib-based induction treatment before autologous stem-cell transplantation in patients with previously untreated multiple myeloma: a meta-analysis of phase III randomized, controlled trials. J. Clin. Oncol. 31, 3279-3287 (2013). 
34. Durie, B. G. et al. Bortezomib with lenalidomide and dexamethasone versus lenalidomide and dexamethasone alone in patients with newly diagnosed myeloma without intent for immediate autologous stem-cell transplant (SWOG S0777): a randomised, open-label, phase 3 trial. Lancet 389, 519-527 (2017).

35. Roussel, M. et al. Front-line transplantation program with lenalidomide, bortezomib, and dexamethasone combination as induction and consolidation followed by lenalidomide maintenance in patients with multiple myeloma: a phase II study by the Intergroupe Francophone du Myelome. J. Clin. Oncol. 32, 2712-2717 (2014).

36. Rosinol, L. et al. Bortezomib, Lenalidomide and Dexamethasone (VRD-GEM) as induction therapy prior autologous stem cell transplantation (ASCT) in multiple myeloma (MM): results of a prospective phase III Pethema/GEM Trial. Blood 130(Suppl 1), 2017 (2017).

37. Lonial, S. et al. Daratumumab monotherapy in patients with treatmentrefractory multiple myeloma (SIRIUS): an open-label, randomised, phase 2 trial. Lancet 387, 1551-1560 (2016).

38. Costello, C. An update on the role of daratumumab in the treatment of multiple myeloma. Ther. Adv. Hematol. 8, 28-37 (2017).

39. Genmab. Genmab announces positive topline results in Phase III CASSIOPEIA Study of Daratumumab in front line multiple myeloma. https:/ir.genmab. com/news-releases/news-release-details/genmab-announces-positivetopline-results-phase-iii-cassiopeia (2018).

40. Voorhees, P. M. et al. Interim safety analysis of a phase 2 randomized study of Daratumumab (Dara), Lenalidomide $(R)$, Bortezomib $(M)$, and Dexamethasone (d; Dara-RVd) vs. RVd in patients (Pts) with newly diagnosed multiple myeloma (MM) eligible for high-dose therapy (HDT) and autologous stem cell transplantation (ASCT). Blood 130(Suppl 1), 1879 (2017).

41. Voorhees, P. M. et al. Efficacy and updated safety analysis of a safety run-in cohort from Griffin, a phase 2 randomized study of Daratumumab (Dara), Bortezomib (V), Lenalidomide (R), and Dexamethasone (D; Dara-Vrd) vs. Vrd in patients (Pts) with newly diagnosed (ND) multiple myeloma (MM) eligible for high-dose therapy (HDT) and autologous stem cell transplantation (ASCT). Blood 132(Suppl 1), 151 (2018).

42. Yimer, $\mathrm{H}$. et al. Lyra: a phase 2 study of Daratumumab (Dara) plus Cyclophosphamide, Bortezomib, and Dexamethasone (Cybord) in newly diagnosed and relapsed patients (Pts) with multiple myeloma (MM). Blood, 132 (Suppl 1), 152 (2018).

43. Andrzej, J. et al. Daratumumab (DARA) in combination with Carfilzomib, Lenalidomide, and Dexamethasone (KRd) in patients (pts) with newly diagnosed multiple myeloma (MMY1001): an open-label, phase 1b study. J. Clin. Oncol. 35, 8000-8000 (2017)

44. Cavo, M. et al. International Myeloma Working Group consensus approach to the treatment of multiple myeloma patients who are candidates for autologous stem cell transplantation. Blood 117, 6063-6073 (2011).

45. Moreau, P., Attal, M. \& Facon, T. Frontline therapy of multiple myeloma. Blood 125, 3076-3084 (2015).

46. Anderson, K. C. et al. Multiple myeloma, Version 2.2016: clinical practice guidelines in oncology. J. Natl. Compr. Canc. Netw. 13, 1398-1435 (2015).

47. Gertz, M. A. \& Dingli, D. How we manage autologous stem cell transplantation for patients with multiple myeloma. Blood 124, 882-890 (2014).

48. Moreau, P. et al. Achievement of VGPR to induction therapy is an important prognostic factor for longer PFS in the IFM 2005-01 trial. Blood 117, 3041-3044 (2011).

49. Binder, M. et al. Predictors of early response to initial therapy in patients with newly diagnosed symptomatic multiple myeloma. Am. J. Hematol. 90, 888-891 (2015).

50. Avet-Loiseau, H. L.-C. V. et al. Minimal residual disease in multiple myeloma: final analysis of the IFM2009 trial. Abstract \#435. In Presented at the 2017 American Society of Hematology Annual Meeting, 10 December, 2017, Atlanta, GA (2017).

51. Ludwig, H. et al. Randomized phase II study of bortezomib, thalidomide, and dexamethasone with or without cyclophosphamide as induction therapy in previously untreated multiple myeloma. J. Clin. Oncol. 31 247-255 (2013).

52. Kumar, S. et al. Randomized, multicenter, phase 2 study (EVOLUTION) of combinations of bortezomib, dexamethasone, cyclophosphamide, and lenalidomide in previously untreated multiple myeloma. Blood 119, 4375-4382 (2012).
53. Sonneveld, P. et al. Phase 2 study of carfilzomib, thalidomide, and dexamethasone as induction/consolidation therapy for newly diagnosed multiple myeloma. Blood 125, 449-456 (2015).

54. Jakubowiak, A. J. et al. A phase $1 / 2$ study of carfilzomib in combination with lenalidomide and low-dose dexamethasone as a frontline treatment for multiple myeloma. Blood 120, 1801-1809 (2012).

55. Mohty, M. \& Ho, A. D. In and out of the niche: perspectives in mobilization of hematopoietic stem cells. Exp. Hematol. 39, 723-729 (2011).

56. Mohty, M. et al. The role of plerixafor in optimizing peripheral blood stem cell mobilization for autologous stem cell transplantation. Leukemia $\mathbf{2 5}, 1-6$ (2011).

57. Bensinger, W., DiPersio, J. F. \& McCarty, J. M. Improving stem cell mobilization strategies: future directions. Bone Marrow Transplant. 43, 181-195 (2009).

58. Larsen, S. R., Chng, K., Battah, F., Martiniello-Wilks, R. \& Rasko, J. E. Improved granulocyte colony-stimulating factor mobilization of hemopoietic progenitors using cytokine combinations in primates. Stem Cells 26, 2974-2980 (2008).

59. Sheppard, D., Bredeson, C., Allan, D. \& Tay, J. Systematic review of randomized controlled trials of hematopoietic stem cell mobilization strategies for autologous transplantation for hematologic malignancies. Biol. Blood Marrow Transplant. 18, 1191-1203 (2012).

60. Desikan, K. R. et al. Comparable engraftment kinetics following peripheralblood stem-cell infusion mobilized with granulocyte colony-stimulating factor with or without cyclophosphamide in multiple myeloma. J. Clin. Oncol. 16, 1547-1553 (1998).

61. Russell, N. et al. Plerixafor and granulocyte colony-stimulating factor for firstline steady-state autologous peripheral blood stem cell mobilization in lymphoma and multiple myeloma: results of the prospective PREDICT trial. Haematologica 98, 172-178 (2013).

62. de Mel, S. et al. Vinorelbine-Cyclophosphamide compared to Cyclophosphamide in peripheral blood stem cell mobilization for multiple myeloma. Hematol. Oncol. Stem Cell Ther. 11, 225-232 (2018).

63. Gettys, S. C. et al. Modified CVAD and modified CBAD compared to highdose cyclophosphamide for peripheral blood stem cell mobilization in patients with multiple myeloma. Eur. J. Haematol. 98, 388-392 (2017).

64. Czerw, T. et al. Increased efficacy of stem cell chemo-mobilization with intermediate-dose cytarabine plus G-CSF compared with G-CSF alone in patients with multiple myeloma: results of a randomized trial. Biol. Blood Marrow Transplant. https://doi.org/10.1016/j.bbmt.2018.09.023 (2018).

65. Kumar, S. et al. Impact of lenalidomide therapy on stem cell mobilization and engraftment post-peripheral blood stem cell transplantation in patients with newly diagnosed myeloma. Leukemia 21, 2035-2042 (2007).

66. Mazumder, A. et al. Effect of lenalidomide therapy on mobilization of peripheral blood stem cells in previously untreated multiple myeloma patients. Leukemia 22, 1280-1281 (2008). author reply1-2.

67. Li, S., Fu, J., Ma, H., Mapara, M. Y. \& Lentzsch, S. Lenalidomide-induced upregulation of CXCR4 in CD34+ hematopoietic cells, a potential mechanism of decreased hematopoietic progenitor mobilization. Leukemia 27, 1407-1411 (2013).

68. Silvennoinen, R. et al. A randomized phase II study of stem cell mobilization with cyclophosphamide+G-CSF or G-CSF alone after lenalidomide-based induction in multiple myeloma. Bone Marrow Transplant. 51, 372-376 (2016).

69. Malard, F. et al. Plerixafor for autologous peripheral blood stem cell mobilization in patients previously treated with fludarabine or lenalidomide. Biol. Blood. Marrow Transplant. 18, 314-317 (2012).

70. Wang, G. et al. Recombinant human thrombopoietin improves the efficacy of intermediate-dose cyclophosphamide plus granulocyte colonystimulating factor in mobilizing peripheral blood stem cells in patients with multiple myeloma: a cohort study. Medicine (Baltimore) 96, e9302 (2017).

71. Lahuerta, J. J. et al. Busulfan $12 \mathrm{mg} / \mathrm{kg}$ plus melphalan $140 \mathrm{mg} / \mathrm{m}^{2}$ versus melphalan $200 \mathrm{mg} / \mathrm{m}^{2}$ as conditioning regimens for autologous transplantation in newly diagnosed multiple myeloma patients included in the PETHEMAVGEM2000 study. Haematologica 95, 1913-1920 (2010).

72. Blanes, M. et al. Intravenous busulfan and melphalan as a conditioning regimen for autologous stem cell transplantation in patients with newly diagnosed multiple myeloma: a matched comparison to a melphalan-only approach. Biol. Blood Marrow Transplant. 19, 69-74 (2013).

73. Qazilbash, M. H. et al. A randomized phase III trial of Busulfan+Melphalan vs Melphalan alone for multiple myeloma. Blood 130(Suppl 1), 399 http://www. bloodjournal.org/content/130/Suppl_1/399. Accepted 20 Dec 2018. (2017). 
74. Moreau, P. et al. Melphalan $220 \mathrm{mg} / \mathrm{m}^{2}$ followed by peripheral blood stem cell transplantation in 27 patients with advanced multiple myeloma. Bone Marrow Transplant. 23, 1003-1006 (1999).

75. Bensinger, W. I. et al. A randomized study of melphalan $200 \mathrm{mg} / \mathrm{m}(2)$ vs 280 $\mathrm{mg} / \mathrm{m}(2)$ as a preparative regimen for patients with multiple myeloma undergoing auto-SCT. Bone Marrow Transplant. 51, 67-71 (2016).

76. Hari, P. et al. Final outcomes of escalated melphalan $280 \mathrm{mg} / \mathrm{m}(2)$ with amifostine cytoprotection followed autologous hematopoietic stem cell transplantation for multiple myeloma: high CR and VGPR rates do not translate into improved survival. Bone Marrow Transplant. 54, 293-299 (2019).

77. Roussel, M. et al. Bortezomib and high-dose Melphalan vs. high-dose melphalan as conditioning regimen before autologous stem cell transplantation in de novo multiple myeloma patients: a phase 3 study of the Intergroupe Francophone Du Myelome (IFM 2014-02). Blood 130(Suppl 1), 398 (2017).

78. Farag, S. et al. Dose-intensified bendamustine and melphalan (BenMel) conditioning before second autologous transplantation in myeloma patients. Hematol. Oncol. 36, 671-678 (2018).

79. Martino, M. et al. A phase II, single-arm, prospective study of bendamustine plus melphalan conditioning for second autologous stem cell transplantation in de novo multiple myeloma patients through a tandem transplant strategy. Bone Marrow Transplant. 51, 1197-1203 (2016).

80. Sonneveld, P. et al. Consolidation Followed by Maintenance vs Maintenance Alone in Newly Diagnosed, Transplant Eligible Multiple Myeloma: A Randomized Phase 3 Study of Tte European Myeloma Network (Emn02/Ho95 Mm Trial). 214488 (EHA Learning Center Sonneveld P, 2018).

81. Stadtmauer, E. A. et al. Comparison of autologous hematopoietic cell transplant (autoHCT), Bortezomib, Lenalidomide (Len) and Dexamethasone (RVD) consolidation with Len maintenance (ACM), tandem Autohct with Len maintenance (TAM) and Autohct with Len maintenance (AM) for up-front treatment of patients with multiple myeloma (MM): primary results from the randomized phase III trial of the Blood and Marrow Transplant Clinical Trials Network (BMT CTN 0702-StaMINA Trial). Blood 128, LBA-1 (2016).

82. Paiva, B. et al. High-risk cytogenetics and persistent minimal residual disease by multiparameter flow cytometry predict unsustained complete response after autologous stem cell transplantation in multiple myeloma. Blood $\mathbf{1 1 9}$ 687-691 (2012).

83. Morgan, G. J. et al. The role of maintenance thalidomide therapy in multiple myeloma: MRC Myeloma IX results and meta-analysis. Blood 119, 7-15 (2012).

84. Ludwig, $\mathrm{H}$. et al. IMWG consensus on maintenance therapy in multiple myeloma. Blood 119, 3003-3015 (2012).

85. Stewart, A. K. et al. A randomized phase 3 trial of thalidomide and prednisone as maintenance therapy after ASCT in patients with MM with a quality-of-life assessment: the National Cancer Institute of Canada Clinicals Trials Group Myeloma 10 Trial. Blood 121, 1517-1523 (2013).

86. Spencer, A. et al. Consolidation therapy with low-dose thalidomide and prednisolone prolongs the survival of multiple myeloma patients undergoing a single autologous stem-cell transplantation procedure. J. Clin. Oncol. 27, 1788-1793 (2009).

87. Attal, M. et al. Lenalidomide maintenance after stem-cell transplantation for multiple myeloma. N. Engl. J. Med. 366, 1782-1791 (2012).

88. McCarthy, P. L. et al. Lenalidomide after stem-cell transplantation for multiple myeloma. N. Engl. J. Med. 366, 1770-1781 (2012).

89. McCarthy, P. L. et al. Lenalidomide maintenance after autologous stem-cell transplantation in newly diagnosed multiple myeloma: a meta-analysis. J. Clin. Oncol. 35, 3279-3289 (2017).

90. Jackson, $\mathrm{G}$. et al. Lenalidomide maintenance significantly improves outcomes compared to observation irrespective of cytogenetic risk: results of the Myeloma XI Trial. Blood 130(Suppl 1), 436 (2017).

91. Patel, K. K. et al. Update on a phase II study of Ixazomib with Lenalidomide as maintenance therapy following autologous stem cell transplant in patients with multiple myeloma. Blood 130(Suppl 1), 437 (2017).

92. Dimopoulos, M. A. et al. Maintenance therapy with the oral proteasome inhibitor (PI) Ixazomib significantly prolongs progression-free survival (PFS) following autologous stem cell transplantation (ASCT) in patients with newly diagnosed multiple myeloma (NDMM): Phase 3 Tourmaline-MM3 Trial. Blood 132(Suppl 1), 301 (2018).

93. Barlogie, B. et al. Superiority of tandem autologous transplantation over standard therapy for previously untreated multiple myeloma. Blood $\mathbf{8 9}$ 789-793 (1997).

94. Attal, M. et al. Single versus double autologous stem-cell transplantation for multiple myeloma. N. Engl. J. Med. 349, 2495-2502 (2003).

95. Barlogie, B. et al. Incorporating bortezomib into upfront treatment for multiple myeloma: early results of total therapy 3. Br. J. Haematol. 138, 176-185 (2007).

96. Mai, E. K. et al. Single versus tandem high-dose melphalan followed by autologous blood stem cell transplantation in multiple myeloma: long-term results from the phase III GMMG-HD2 trial. Br. J. Haematol. 173, 731-741 (2016)

97. Cavo, M. et al. Upfront single versus double autologous stem cell transplantation for newly diagnosed multiple myeloma: an intergroup, multicenter, phase III study of the European Myeloma Network (EMN02/HO95 MM Trial). Blood 128, 991 (2016).

98. Cavo, M. et al. Double autologous stem cell transplantation significantly prolongs progression-free survival and overall survival in comparison with single autotransplantation in newly diagnosed multiple myeloma: an analysis of phase 3 EMN02/H095 study. Blood, 130(Suppl 1), 401 (2017).

99. Palumbo, A. et al. Autologous transplantation and maintenance therapy in multiple myeloma. N. Engl. J. Med. 371, 895-905 (2014).

100. Gay, F. et al. Chemotherapy plus lenalidomide versus autologous transplantation, followed by lenalidomide plus prednisone versus lenalidomide maintenance, in patients with multiple myeloma: a randomised, multicentre, phase 3 trial. Lancet Oncol. 16, 1617-1629 (2015).

101. Fermand, J. P. et al. High-dose therapy and autologous peripheral blood stem cell transplantation in multiple myeloma: up-front or rescue treatment? Results of a multicenter sequential randomized clinical trial. Blood 92 3131-3136 (1998)

102. Richardson, P. G., Laubach, J. P., Munshi, N. C. \& Anderson, K. C. Early or delayed transplantation for multiple myeloma in the era of novel therapy: does one size fit all? Hematol Am. Soc. Hematol. Educ. Program 2014, 255-261 (2014).

103. Giralt, S. et al. American Society of Blood and Marrow Transplantation, European Society of Blood and Marrow Transplantation, Blood and Marrow Transplant Clinical Trials Network, and International Myeloma Working Group Consensus Conference on Salvage Hematopoietic Cell Transplantation in Patients with Relapsed Multiple Myeloma. Biol. Blood. Marrow Transplant. 21, 2039-2051 (2015).

104. Cook, G. et al. The effect of salvage autologous stem-cell transplantation on overall survival in patients with relapsed multiple myeloma (final results from BSBMT/UKMF Myeloma X Relapse [Intensive]): a randomised, open-label, phase 3 trial. Lancet Haematol. 3, e340-e351 (2016).

105. Atanackovic, D. \& Schilling, G. Second autologous transplant as salvage therapy in multiple myeloma. Br. J. Haematol. 163, 565-572 (2013).

106. Mateos, M. V. et al. Daratumumab plus Bortezomib, Melphalan, and Prednisone for untreated myeloma. N. Engl. J. Med. 378, 518-528 (2018).

107. Thierry Facon, S. K. K. et al. Phase 3 randomized study of Daratumumab plus Lenalidomide and Dexamethasone (D-Rd) versus Lenalidomide and Dexamethasone (Rd) in patients with newly diagnosed multiple myeloma (NDMM) ineligible for transplant (MAIA). In ASH Annual Meeting Abstracts, Late-Breaking Abstracts Session, LBA-2 (2018).

108. Miller, K. C. et al. Comparable outcomes using propylene glycol-free melphalan for autologous stem cell transplantation in multiple myeloma. Bone Marrow Transplant. 54, 587-594 (2019).

109. Paiva, B. P. N. et al. Impact of next-generation flow (NGF) minimal residual disease (MRD) monitoring in multiple myeloma (MM): results from the Pethema/GEM2012 Trial. Blood, ASH Annual Meeting Abstracts, Vol. 130, Suppl.1, 905 (2017)

110. Perrot, A. et al. Minimal residual disease negativity using deep sequencing is a major prognostic factor in multiple myeloma. Blood 132, 2456-2464 (2018). 